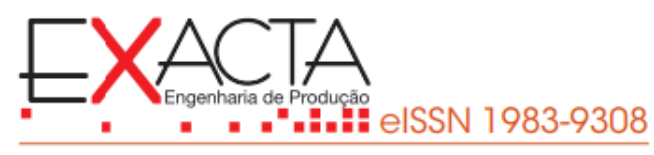

\title{
INFLUÊNCIA DA PRIORIZAÇÃO DE VEÍCULOS MENOS POLUENTES NOS CUSTOS COM COMBUSTÍVEL
}

\section{INFLUENCE OF PRIORIZATION OF LESS POLLUTING VEHICLES ON FLEET FUEL EXPENSES}

Recebido em: 22 out. 2020

Aprovado em: $12 \mathrm{dez} 2020$

Versão do autor aceita publicada online: $12 \mathrm{dez} 2020$

Publicado online: 25 jun. 2021

Como citar esse artigo - American Psychological Association (APA):

Santana, M., \& Oiko, O. T. (2022, out./dez.). Influência da priorização de veículos menos poluentes nos custos com combustível. Exacta. 20(4), 861-883.

https://doi.org/10.5585/exactaep.2022.18554.

Submeta seu artigo para este periódico $\bigoplus$

Dados Crossmark 


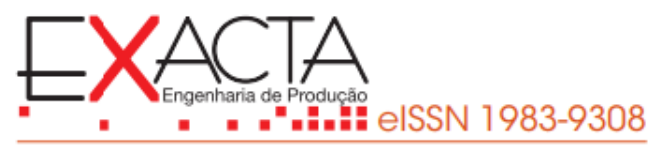

\title{
INFLUÊNCIA DA PRIORIZAÇÃO DE VEÍCULOS MENOS POLUENTES NOS CUSTOS COM COMBUSTÍVEL
}

\author{
INFLUENCE OF PRIORIZATION OF LESS POLLUTING VEHICLES ON FLEET FUEL \\ EXPENSES
}

\author{
${ }^{1}$ Mestrado \\ Universidade Federal de São Carlos - UFSCar. \\ São Carlos, SP - Brasil \\ mateus.95.santana@gmail.com \\ 2 Doutorado \\ Universidade Estadual de Maringá - UEM. \\ Maringá, PR - Brasil. \\ otoiko@uem.br
}

Resumo: A atividade de transporte contribui significativamente para agravar os impactos ambientais das atividades logísticas e, portanto, um forte objeto de estudo da Logística Verde. Neste estudo, foi analisada a pegada de carbono (CFP) de uma frota empresarial durante 12 meses, utilizando-se o método do GHG Protocol. Por observar que o custo pudesse ser uma barreira para a empresa adotar políticas que priorizem a redução de seus impactos ambientais, este trabalho investigou quão oneroso seria priorizar o uso de combustíveis e veículos menos poluentes. Assim, propôs-se um cenário em que se buscou minimizar os valores da CFP de tais viagens e, em outro, os gastos com combustível. Finalmente, comparou-se os impactos ambientais e financeiros entre os cenários por meio das métricas: Custo Incremental e Incremento Percentual em relação ao cenário de menor impacto ambiental. Foi possível mensurar a variação da CFP e dos custos financeiros, fornecendo à empresa embasamento para a tomada de decisão visando minimizar os impactos ambientais.

Palavras-chave: Logística Verde. Pegada de Carbono. Ecologística. GHG Protocol. Emissões Atmosféricas.

Abstract: Transportation contributes significantly to aggravate the environmental impacts of logistics activities and, therefore, is a strong object of study for Green Logistics. Here, the Carbon Footprint (CFP) of a company's fleet was analyzed for 12 months using the GHG Protocol method. After observing that cost could be a barrier to company's adoption of policies that reduces its environmental impacts, this study investigated how costly it would be to prioritize the use of less polluting fuels and vehicles. Thus, a scenario was proposed in which it sought to minimize the CFP values of such trips and, in another one, fuel costs. Finally, the environmental and financial impacts between the scenarios were compared through two metrics: Increase of Cost and Percentage Increase in relation to the lowest environmental impact scenario. It was possible to measure the variation of the CFP and fuel costs, providing the company a basis for decision making in order to minimize their environmental impacts. 
1 Introdução

A temperatura da superfície da Terra tem aumentado consideravelmente nas últimas três décadas desde 1850, segundo o Painel Intergovernamental de Mudanças Climáticas [IPCC] (2014). Tal aumento pode estar associado a diversas consequências negativas ao planeta, como a alteração de ciclos hidrológicos, acidificação dos oceanos e mudanças no comportamento de diversas espécies de animais. Na América do Sul, por exemplo, é muito provável que esse aquecimento tenha impactado negativamente os ciclos de precipitações de chuvas e neve, os ecossistemas marinhos e até mesmo a produção de alimentos (IPCC, 2014).

Ainda nesse relatório, o IPCC afirma ser extremamente provável que mais da metade do aumento observado da temperatura média global da superfície terrestre entre 1951 e 2010 ocorreu devido ao aumento antropogênico das concentrações de gases do efeito estufa (GEE) na atmosfera, em especial do $\mathrm{CO}_{2}, \mathrm{NH}_{4}$ e $\mathrm{N}_{2} \mathrm{O}$. As emissões de tais gases se intensificaram substancialmente nos últimos 40 anos, de forma que mais da metade da quantidade emitida entre 1750 e 2011 ocorreu apenas nesse período, conforme a Figura 1 ilustra (IPCC, 2014):

\section{Figura 1}

Emissões Globais Antropogênicas de $\mathrm{CO}_{2}$ Provenientes da Silvicultura e Outros Usos da Terra, Bem Como da Queima de Combustíveis Fósseis, Produção de Cimento e Queimas
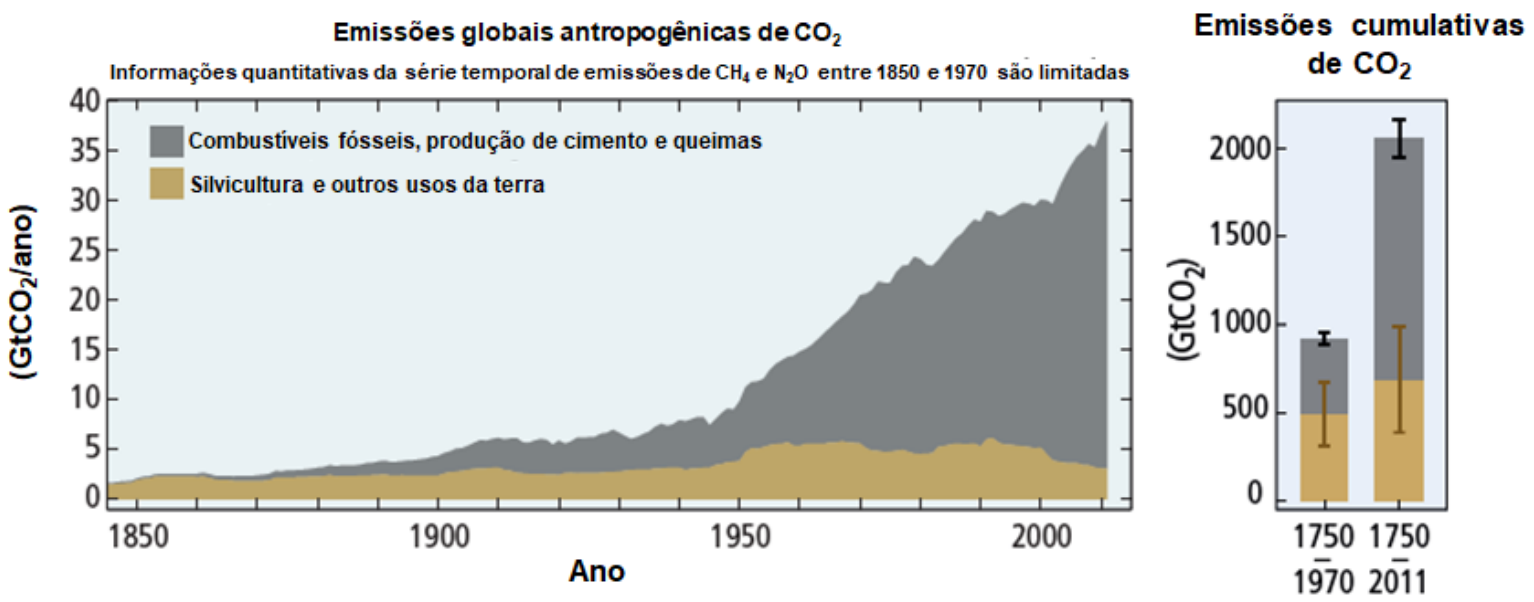

Fonte: IPCC, p.3, 2014

Ao se analisar as fontes de tais emissões, observa-se que o setor de Energia é responsável por cerca de $90 \%$ das emissões de $\mathrm{CO}_{2}$ e $75 \%$ das emissões totais de GEE em países desenvolvidos. Desse montante, cerca de $25 \%$ são provenientes de combustões móveis, ou seja, atividades de transporte (IPCC, 2006). Tais números revelam a magnitude dos impactos ambientais na atmosfera resultantes das operações de transporte, componente da Logística que mais degrada o meio ambiente (Wolf \& Seuring, 
2010). De fato, há uma área de estudo dentro da Logística chamada Logística Verde que se preocupa justamente com seus aspectos ambientais (Donato, 2008), a qual será melhor explanada na Seção 2.1.

O meio empresarial já há mais de duas décadas reconhecia a relevância da problemática e a necessidade de se resolvê-la. Murphy, Poist e Braunschweig (1995) realizaram uma pesquisa com 133 gestores da área de logística e membros do Council of Logistics Management (atual Council of Supply Chain Management), dos quais $60,2 \%$ declaravam que fatores ambientais eram altamente importantes às suas empresas e $85,2 \%$ que a importância dada pelas mesmas ao tema nos próximos 5 anos aumentaria. Porém, de acordo com Winsemius e Guntram (1992), o foco das empresas em relação a tais questões naquela época era majoritariamente cumprir com a legislação, sendo a integração da preservação ambiental às suas estratégias uma das menores prioridades. Atualmente, segundo Walker, Di Sisto e Mc Bain (2008), além das regulamentações governamentais, as principais motivações para maior preocupação ambiental pelas empresas na gestão de sua cadeia de suprimentos se devem também às pressões por parte dos consumidores e da sociedade - ambos mais conscientes dos problemas ambientais e do papel do setor privado em relação aos mesmos, e às empresas concorrentes por obterem maior vantagem competitiva ao adotarem políticas voltadas ao meio ambiente. Contudo, ainda segundo os mesmos autores, a principal barreira interna das empresas para adoção de estratégias voltadas ao meio ambiente são os custos gerados por estas.

Na literatura científica, observou-se um crescimento nos trabalhos acerca do tema ao longo das últimas décadas. Seuring e Müller (2008), em uma revisão da literatura sobre cadeias de suprimentos sustentáveis, identificaram poucos trabalhos publicados sobre o tema em periódicos com foco em gestão entre 1994 e 1999, com crescimento relativamente alto a partir dos anos 2000. Santos, Bortolon, Chiroli e Oiko (2015) realizaram uma revisão sistemática sobre Logística Verde, na qual encontraram pouco artigos sobre o tema entre 1994 e 2006, com aumento de publicações entre 2007 e 2010 e um crescimento considerável de 2011 a 2013. Já na revisão sistemática de Silva, Vaz e Lezana (2020) sobre Green Supply Managment e Logística Reversa, foram observadas diversas lacunas de pesquisa que possuem intrínsecas relações com a Logística Verde, como o desenvolvimento de métodos para avaliação de desempenho ambiental e de medidas para prevenção de danos ambientais ao longo do ciclo de vida de novos produtos, bem como a necessidade de buscar eficiência para reduzir os custos.

Porém, segundo Meixell e Norbis (2008), nos trabalhos específicos sobre tomada de decisão de meios de transporte, os critérios relacionados a impactos ambientais e uso de energia até 2008 ainda não eram levados em consideração com frequência pelos autores.

Dentre as ações já existentes para a redução da degradação antropogênica do meio ambiente, uma delas foi a criação da Pegada de Carbono (ou Carbon Footprint, CFP), indicador ambiental que, embora não tenha uma definição unânime na literatura, possibilita mensurar os impactos ambientais de empresas e organizações e definir planos de ação para minorá-los, podendos ser particularizado às 
atividades de transporte como observado em alguns trabalhos da literatura: Kellner e |g| (2015) utilizaram a CFP para avaliar como a forma como a rede de distribuição de um operador logístico se configura pode alterar consideravelmente os impactos ambienteis gerados por uma mesma entrega; Duez (2016), Zabeo, Bellio, Pizzol, Giubilato e Semenzin (2017), Saenz, Figliozzi e Faulin (2016) empregaram a CFP para analisar a redução das emissões de GEE de veículos ao substituir, respectivamente: o modelo atual de pneus de uma frota de caminhões por um novo específico, motores à diesel por a gás natural e à diesel por elétricos; Hao, Geng e Sarkis (2016) avaliaram o quanto a atmosfera será poluída por veículos de passeio até 2050 ao prever a variação da CFP global destes; e Silva, Ferreira e Steiner (2019) avaliaram o uso de métodos de otimização exatos e heurísticos clássicos da Pesquisa Operacional para definição de rotas de ônibus sob uma ótica ambiental, minimizando as emissões de GEE resultantes.

Neste estudo, foram analisadas as Pegadas de Carbono das viagens realizadas pela frota baixa de um abatedouro durante o período de 12 meses. Como os custos são uma preocupação constante das empresas e também uma barreira comum à adoção de práticas que melhoram o desempenho ambiental, este trabalho também investigou a quão onerosa tal proposta menos poluente seria em relação tanto ao histórico real de dados quanto à proposta mais econômica para os trajetos percorridos. Para isto, propôs-se a incorporação da CFP como critério na decisão de alocação de frota para cada viagem e simulou-se a variação do custo e das emissões de GEE após a incorporação. Ao final das simulações, foi possível responder: "qual o impacto financeiro para a empresa causado pela minimização da Pegada de Carbono na alocação de veículos da frota baixa?"

Na seção 2, é apresentado o referencial teórico sobre o método e os argumentos utilizados na discussão; na seção 3, são descritos os parâmetros utilizados para análises e seus referidos métodos de cálculo; na seção 4 encontra-se a caracterização do caso estudado e, na seção 5, são apresentados os resultados dos cálculos e as interpretações realizadas com base nos mesmos.

\section{Referencial teórico}

Dado o objetivo deste trabalho, são apresentadas nas seções seguintes as definições de Logística Verde e CFP, relevantes para o estudo realizado.

\subsection{Logística verde}

A Logística é constituída por diversas atividades, sendo as principais: transporte, gerência de estoque e processamento de pedidos. Essas atividades são consideradas principais devido à sua larga contribuição para o custo total das operações logísticas e por serem essenciais para a coordenação e o cumprimento das tarefas logísticas (Ballou, 2006). 
Do ponto de vista ecológico, Donato (2008) afirma que grande parte da poluição ambiental verificada nos grandes centros urbanos é causada predominantemente pelos poluentes atmosféricos gerados pela queima de combustíveis fósseis em veículos automotores. Dessa forma, observa-se que as atividades de transporte e a forma como são gerenciadas pela empresa podem interferir significantemente nos efeitos negativos gerados pela Logística sobre o meio ambiente.

A área da Logística que trata de suas consequências ambientais é denominada de Logística Verde ou Ecologística (Donato, 2008). No âmbito desta, avaliam-se os impactos das operações relacionadas ao armazenamento, manejo e transporte de mercadorias pela cadeia de produção, analisando sua natureza e gravidade e buscando meios de minorá-las (Mckinnon, Browne, Whiteing \& Piecyk, 2010).

O maior intuito da logística verde é articular as operações em uma cadeia de suprimentos de maneira que os requisitos dos beneficiários sejam satisfeitos reduzindo os danos ambientais. Ao avaliar sistemas de produção sob a ótica deste conceito, o "custo", previamente considerado em termos apenas financeiros, passa também a incluir a degradação ambiental resultante dessas atividades: alterações climáticas, poluição do ar, deposição de resíduos (incluindo os resíduos de embalagens), degradação do solo, ruído, vibração e acidentes [...] (Quiumento, 2011 como citado em Santos et al., 2015, p. 315).

Dentre as principais consequências negativas dessa degradação ambiental, pode-se citar: odor, ruído, resíduos sólidos, contaminação de efluentes, fumaça e material radioativo, sendo a fumaça um dos poluentes mais prejudiciais (Donato, 2008).

Neste cenário, nota-se a importância de se tomar medidas para a redução da poluição atmosférica gerada pelas atividades de transporte. Porém, antes de definir quais medidas tomar, é essencial haver uma forma de quantificar a poluição. Dada a dificuldade de medições diretas da quantidade exata de poluentes emitida por determinado modal enquanto em atividade, uma solução seria a mensuração indireta por meio da Pegada de Carbono, a qual será explicada na próxima seção.

\subsection{Pegada de carbono}

Wieldman e Minx (2008) discutiram as definições para o termo Carbon Footprint, e propuseram uma nova definição, utilizada largamente em trabalhos seguintes. Já naquele ano existiam diversos significados para a expressão e métodos de se calculá-la. No tocante especificamente às atividades de transporte, Santana e Oiko (2019) realizaram uma revisão sistemática da literatura sobre o tema, evidenciando a variedade de conceitos atribuídos a tal termo e comparando-os quanto aos seus escopos de aplicação, gases poluentes considerados e métodos de cálculo. A seguir, são apresentadas algumas dessas definições. 
Wiedman e Minx (2008) definiram "Pegada de Carbono" como "(...) uma medida do montante total e exclusivo de $\mathrm{CO}_{2}$ que é gerado direta e indiretamente por uma atividade ou que é acumulado durante os estágios de vida de um produto" (Wiedman \& Minx, 2008, p. 4). Quanto aos métodos de cálculo, os autores trouxeram duas abordagens diferentes, porém com o mesmo objetivo: realizar uma Análise de Ciclo de Vida (ACV) completa. Uma das abordagens é a chamada Process Analysis, a qual estuda os impactos ambientais de cada produto do berço ao túmulo (craddle to grave). A outra é denominada Environmental input-output, detalhada em Wiedman e Minx (2008). Wiedman e Minx (2008) ainda explicam que, para uma análise detalhada, o ideal é combinar ambos os métodos por meio de uma abordagem híbrida, descrita em Bullard, Penner e Pilati (1978).

A norma ISO14067:2013 traz especificações técnicas as quais detalham princípios, requerimentos e diretrizes para a quantificação e comunicação da pegada de carbono total e parcial de um produto, incluindo tanto bens quanto serviços, baseado em suas emissões e sequestros de GEE durante o ciclo de vida do produto em estudo (International Organization for Standardization [ISO], 2013). Segundo a ISO 14067:2013, a definição da pegada de carbono de um produto é: "soma das emissões e sequestros de GEE que ocorrem durante o ciclo de vida de um produto, expressa em dióxido de carbono equivalente $\left(\mathrm{CO}_{2 \text { eq }}\right)$ e baseada em sua $\mathrm{ACV}$ utilizando a categoria de impacto de mudanças climática" (ISO, 2013, p. 2). Também é apresentada a definição de pegada de carbono parcial: "soma das emissões e sequestros de GEE de um ou mais processos selecionados do ciclo de vida de um produto, expressa em $\mathrm{CO}_{2 \text { eq }}$ e baseado nos estágios ou processos relevantes em seu ciclo de vida" (ISO, 2013, p. 2). Para a realização do cálculo da pegada de carbono, a norma não descreve um método específico, mas sim diretrizes e normas que devem ser seguidas para cálculo e documentação dos resultados. Assim, um estudo de pegada de carbono, de acordo com as normas técnicas dessa ISO, deve incluir as quatro fases da ACV (definição do objetivo e do escopo, inventário de ciclo de vida, avaliação do impacto do ciclo de vida e interpretação do ciclo de vida (ISO, 2013).

Outra definição é apresentada pela Global Footprint Network (GFN), organização sem fins lucrativos fundada em 2003 que desenvolve estudos e ferramentas que contribuem para o desenvolvimento sustentável, sendo reconhecida internacionalmente por diversos prêmios. A organização desenvolve estudos voltados para a pegada ecológica (Ecological Footprint - EF), cujo conceito é: "A mensuração dos ativos ecológicos que determinada população demanda para produzir os recursos naturais que consome e para absorver seus resíduos, principalmente os que são em forma de emissões de carbono" (GFN, 2017, p. 1). Segundo a organização, a CFP é um dos componentes da EF, representando atualmente cerca de 60\% de seu valor. Quanto à definição específica de CFP, GFN (2017) definiu como o montante de área produtiva (terra e mar) requerida para sequestrar as emissões de $\mathrm{CO}_{2}$ geradas por uma organização ou atividade. Em seu site, é disponibilizada uma calculadora para a realização dos cálculos da EF, cujo método está descrito em Borucke et al. (2013). 
Outra definição para CFP é dada pela Carbon Trust, entidade sem fins lucrativos com a missão de acelerar o movimento para uma economia baseada na mínima emissão de carbono, oferecendo suporte técnico para empresas e para o setor público auxiliando na economia de energia, diminuição de emissões de carbono e comercialização de tecnologias limpas (Carbon Trust, 2012). A instituição define CFP como:

\begin{abstract}
(...) emissão total de gases do efeito estufa (GEE) causada direta ou indiretamente por um indivíduo, organização, evento ou produto, e é expressa como dióxido de carbono equivalente $\left(\mathrm{CO}_{2 \mathrm{e}}\right)$. Uma pegada de carbono considera os seis $\mathrm{GEE}$ segundo o protocolo de Kyoto: dióxido de carbono $\left(\mathrm{CO}_{2}\right)$, metano $\left(\mathrm{CH}_{4}\right)$, óxido nitroso $\left(\mathrm{N}_{2} \mathrm{O}\right)$, hidrofluorcarbonetos (HFCs), perfluorocarbonetos (PFCs) e hexafluoreto de enxofre (SF6). (Carbon Trust, 2012, p.2).
\end{abstract}

A organização também sugere dois tipos de CFP.

- Pegada de Carbono Organizacional: mensura as emissões de GEE de todas as atividades ao longo da organização analisada, incluindo a energia utilizada nas instalações, processos industriais e veículos da empresa. Para calcular essa forma de CFP, Carbon Trust (2012) recomenda utilizar a metodologia descrita e atualizada pelo World Resource Institute [WRI] (2008);

- Pegada de Carbono do Produto: mensura as emissões de GEE durante toda a vida do produto (bens ou serviços), da extração de matéria-prima e manufatura até seu uso e reuso final, reciclagem ou descarte. Para calcular essa forma de pegada de carbono, Carbon Trust (2012) recomenda utilizar a metodologia descrita pelo The British Standards Institution [BSI] (2011; Carbon Trust, 2012).

Devido à disponibilidade de dados para a aplicação do método e às características do caso estudado, a definição selecionada para este trabalho é a de Pegada de Carbono Organizacional, sugerida pela Carbon Trust (2012). Por consequência, embora existam diversos métodos de mensuração de CFP, neste estudo utilizou-se da metodologia descrita em WRI (2008) conforme recomendado pela instituição.

\title{
2.3 Método de cálculo
}

A mensuração da CFP segundo WRI (2008) consiste em duas etapas: calcular a CFP e o custo financeiro de cada viagem. As seções 2.3.1 até 2.3.4 explicam como os cálculos foram realizados. 


\subsubsection{Cálculo da pegada de carbono - GHG Protocol}

O The Greenhouse Gas Protocol - A Corporate Accounting and Reporting Standard, ou simplesmente GHG Protocol, é uma das ferramentas mais utilizadas hoje por instituições privadas e governamentais para quantificar e estudar suas emissões de poluentes. Lançada em 1998 e revisada em 2004, a ferramenta é compatível com as normas da International Organization for Standardization (ISO) e com as metodologias de quantificação do Painel Intergovernamental sobre Mudança Climática (IPCC), sendo utilizada no Brasil de forma adaptada desde 2008 (WRI, 2008).

Em WRI (2008), encontram-se as diretrizes para a elaboração de um inventário completo de uma empresa ou organização que deseja mensurar suas emissões, considerando todos os setores envolvidos. Tais diretrizes foram seguidas para a elaboração do presente trabalho mesmo se tratando da quantificação da CFP de uma atividade específica de uma empresa.

\subsubsection{Definição dos limites}

O primeiro passo antes de se iniciarem os cálculos de contabilização da CFP consiste em definir os limites da empresa em que os mesmos serão aplicados (WRI, 2008).

O primeiro limite a ser considerado é o limite geográfico. A fim de evitar dupla contabilização de emissões atmosféricas, os inventários devem incluir apenas emissões ocorridas em território brasileiro. Caso haja emissões ocorridas fora do Brasil, as mesmas devem ser indicadas separadamente (WRI, 2008).

Em seguida, deve-se levar em consideração os limites organizacionais da empresa, os quais dependem fortemente de sua estrutura legal. Para defini-los, duas abordagens devem ser utilizadas: controle operacional e participação societária. Por meio da abordagem de controle operacional, uma organização responde por $100 \%$ das emissões de GEE das unidades sobre as quais tem controle das operações, não respondendo por aquelas provenientes das quais tem apenas participação societária. Já na abordagem de participação societária, são contabilizadas as emissões decorrentes das operações considerando a porcentagem de participação da empresa em estudo no capital de tal operação (WRI, 2008). No caso em estudo, uma vez que toda a frota pertence ao abatedouro e todas as atividades logísticas mensuradas foram realizadas por colaboradores do mesmo, utilizou-se apenas a abordagem de controle operacional.

Por fim, definem-se os limites operacionais, processo que envolve a classificação das emissões como diretas ou indiretas e a seleção do escopo para contabilização. A categorização entre emissões diretas ou indiretas está relacionada ao controle ou pertencimento das fontes emissoras: caso pertençam ou sejam controladas pela organização sendo inventariada, são categorizadas como diretas; do contrário, são indiretas. Para aumentar a transparência do inventário e torná-lo mais versátil, 
também é necessário agrupar as fontes emissoras em escopos: o escopo 1 corresponde às fontes de emissões diretas; o escopo 2 contabiliza as emissões provenientes da compra de energia elétrica e térmica; e o escopo 3 contém as formas restantes de emissões indiretas. 0 escopo 1 ainda possui subdivisões em que as fontes devem estar categorizadas: combustões estacionárias; combustões móveis; processos físicos e químicos industriais; emissões fugitivas; e emissões agrícolas (WRI, 2008).

O Protocolo ainda traz uma observação importante quanto às emissões provenientes da combustão de biomassa: tais emissões devem ser segregadas das emissões totais, uma vez que o $\mathrm{CO}_{2}$ liberado na combustão desse material é igual ao $\mathrm{CO}_{2}$ retirado da atmosfera durante o processo de fotossíntese, sendo assim considerado por WRI (2008) como "carbono neutro."

\subsubsection{Etapas para cálculo}

Os passos a serem seguidos para o cálculo das emissões são apresentadas em WRI (2008) e consistem em:

1. Identificar fontes de emissão: Definir os limites operacionais, agrupando as fontes de emissões em seus devidos escopos;

2. Escolher a abordagem de cálculo: Dentre os métodos existentes, o Protocolo menciona a mensuração direta de GEE para cada fonte; balanços de massa ou estequiométricos; ou a aplicação de fatores de emissão documentados, sendo esta a mais frequentemente utilizada;

3. Coletar dados e escolher fatores de emissão;

4. Aplicar ferramentas de cálculo: Apesar de disponibilizar uma planilha eletrônica própria de cálculo, WRI (2008) explica que as organizações podem utilizar ferramentas próprias, desde que as mesmas sejam mais precisas ou estejam de acordo com as diretrizes do Protocolo;

5. Compilar dados no nível corporativo: Processo de compilação de dados das diversas unidades e divisões da empresa a fim de relatar suas emissões totais.

\subsection{Ferramenta de cálculo}

Conforme mencionado anteriormente, o próprio programa GHG Protocol oferece uma ferramenta para a mensuração das emissões em seu site (WRI, 2016). A ferramenta consiste em uma planilha eletrônica já segmentada de acordo com os escopos e as subdivisões impostas pelo Protocolo, além de trazer as fontes bibliográficas dos fatores e parâmetros de cálculo presentes nela.

Para o caso das combustões móveis, ainda há algumas orientações para a utilização da ferramenta. Na planilha, há 3 opções de cálculo para esse tipo de emissões, as quais requerem 
diferentes dados de entradas e possuem diferentes níveis de precisão, conforme é possível observar no Quadro 1:

\section{Quadro 1}

Caracterização das Opções de Cálculo Oferecidas Pela Planilha

\begin{tabular}{|cccc|}
\hline Opção & Parâmetros de Entrada & Parâmetro de Atividade & Nível de Precisão \\
\hline 1 & Tipo da Frota; Ano da Frota; & Consumo de Combustível & - \\
2 & Tipo de Combustível & Consumo de Combustível & Menor que Opção 1 \\
3 & Tipo da Frota; Ano da Frota & Distância Percorrida & Menor que Opção 1 e 2; \\
\hline
\end{tabular}

Fonte: Autoria Própria.

Em todas as opções, são apresentadas ao final as emissões totais por combustão móvel em toneladas de $\mathrm{CO}_{2}$ equivalente $\left(\mathrm{tCO}_{2 \mathrm{e}}\right)$ e, separadamente, as emissões de $\mathrm{CO}_{2}$ biogênico (proveniente de biomassa).

Uma vez que os dados de atividade disponibilizados pela empresa foram as distâncias percorridas em cada viagem, os cálculos de CFP de cada cenário foram realizados utilizando-se a Opção 03 da ferramenta WRI (2016).

As equações 01 e 02 abaixo explicitam o método de cálculo de CFP utilizado pela Opção 03 da planilha:

$$
\begin{aligned}
& F E_{e}=\left[\left(\% F * F E_{C_{2 F}}\right)+\left(F E_{C_{H_{4}}} * G W P_{C_{4}}\right)+\left(F E_{N_{2} O} * G W P_{N_{2} O}\right)\right] * \frac{1}{A u t} \\
& C F P=F E_{e} * \text { Dist }
\end{aligned}
$$

Os valores dos parâmetros são obtidos de diversas fontes: a autonomia média e os fatores de emissão são provenientes do Ministério do Meio Ambiente [MMA] (2014); a porcentagem de combustível fóssil presente em cada combustível foi considerada de acordo com o estabelecido na 
Portaria no 75 de 2015 do Ministério da Agricultura, Pecuária e Abastecimento [MAPA] (2015); e os valores de GWP para cada combustível são dados pelo IPCC (2006).

\section{Procedimentos metodológicos}

\subsection{Objeto de estudo}

Este trabalho foi realizado em uma indústria alimentícia que se encontra ao norte do Paraná, com matriz na cidade de Maringá. A indústria opera principalmente com o abate e processamento de aves, sendo este parcialmente verticalizado. Ainda, a indústria dispõe de filiais em mais oito cidades no mesmo estado.

A frota estudada compõe a denominada "Frota Baixa", um subsetor pertencente ao setor de Transporte da indústria. De acordo com o líder deste, a Frota Baixa é ofertada aos trabalhadores possibilitando que estes se locomovam tanto entre as unidades da firma como para outros pontos, se preciso, por motivos profissionais.

Adicionalmente, limites geográficos, organizacionais e operacionais do objeto de estudo foram definidos de acordo com o método de WRI (2008).

Em relação aos limites geográficos, todas as emissões foram incluídas na contabilização, uma vez que todas os trajetos percorridos pelos veículos da empresa ocorreram integralmente em território nacional.

Sobre os limites organizacionais, uma vez que toda a frota pertence ao abatedouro e todas as atividades logísticas mensuradas foram realizadas pelos colaboradores deste, utilizou-se apenas a abordagem de controle operacional.

Quanto aos limites operacionais, uma vez que as emissões são provenientes somente de fontes pertencentes ao abatedouro, todas se enquadram como emissões diretas de escopo 1 na subcategoria de combustões móveis.

\subsection{Procedimento de coleta de dados}

Os dados de distâncias percorridas e as características dos veículos necessárias para se efetuar os cálculos de custo e CFP foram fornecidos pela empresa conforme explicado nos itens 3.2.1 e 3.2.2 a seguir.

\subsubsection{Registro de atividade dos veículos}

Se um trabalhador precisar de algum veículo, ele necessita solicitá-lo, com, no mínimo, 1 dia de antecedência ao responsável pela Frota Baixa, possibilitando que este disponibilize algum veículo. 
No instante de partida, o responsável registra a "Ficha de Viagem" do veículo, contendo dados como: quilometragem apontada no hodômetro, quantidade de combustível no início e anomalias do veículo. Ao retomar este, são avaliadas as suas condições finais: quilometragem do hodômetro, quantidade de combustível e presença de anomalias externas, exceto as já presentes anteriormente ao percurso.

Finalmente, tais informações são registradas em uma planilha que executa cálculos automáticos que provem indicações importantes ao gerente do setor de Transporte, a exemplo de deterioração e custo por quilômetro rodado.

\subsubsection{Dados de caracterização da frota}

Em relação às características da Frota Baixa, a identificação de cada veículo se dá através de sua respectiva placa e pelo seu número do RENAVAM. No total, foram constatados 90 veículos, segmentados de acordo com suas classificações (7, ao todo), combustíveis (3 tipos, ao todo) e anosmodelos (17, ao todo).

A composição mensal e média da frota ao longo do período estudado pode ser encontrada na tabela 1 a seguir:

Tabela 1

Composição Mensal e Média da Frota Disponível ao Longo de 12 meses.

\begin{tabular}{|lccccccc|}
\hline \multicolumn{1}{|c}{ Mês } & $\begin{array}{c}\text { Automóvel a } \\
\text { gasolina }\end{array}$ & $\begin{array}{c}\text { Automóvel } \\
\text { flex }\end{array}$ & $\begin{array}{c}\text { Comercial } \\
\text { leve } \\
\text { diesel }\end{array}$ & $\begin{array}{c}\text { Comercial } \\
\text { leve a } \\
\text { gasolina }\end{array}$ & $\begin{array}{c}\text { Comercial } \\
\text { leve flex }\end{array}$ & $\begin{array}{c}\text { Veículos } \\
\text { Pesados }\end{array}$ & Motocicleta \\
\hline mar/16 & $4 \%$ & $78 \%$ & $4 \%$ & $4 \%$ & $4 \%$ & $4 \%$ & $4 \%$ \\
abr/16 & $3 \%$ & $77 \%$ & $3 \%$ & $3 \%$ & $10 \%$ & $3 \%$ & $0 \%$ \\
mai/16 & $5 \%$ & $76 \%$ & $10 \%$ & $0 \%$ & $5 \%$ & $5 \%$ & $0 \%$ \\
jun/16 & $4 \%$ & $61 \%$ & $4 \%$ & $4 \%$ & $18 \%$ & $11 \%$ & $0 \%$ \\
jul/16 & $5 \%$ & $75 \%$ & $10 \%$ & $0 \%$ & $10 \%$ & $0 \%$ & $0 \%$ \\
ago/16 & $4 \%$ & $67 \%$ & $4 \%$ & $0 \%$ & $19 \%$ & $7 \%$ & $0 \%$ \\
set/16 & $0 \%$ & $67 \%$ & $0 \%$ & $5 \%$ & $29 \%$ & $0 \%$ & $0 \%$ \\
out/16 & $0 \%$ & $76 \%$ & $0 \%$ & $0 \%$ & $24 \%$ & $0 \%$ & $0 \%$ \\
nov/16 & $0 \%$ & $78 \%$ & $0 \%$ & $0 \%$ & $22 \%$ & $0 \%$ & $0 \%$ \\
dez/16 & $0 \%$ & $84 \%$ & $0 \%$ & $0 \%$ & $16 \%$ & $0 \%$ & $0 \%$ \\
jan/17 & $4 \%$ & $75 \%$ & $0 \%$ & $4 \%$ & $18 \%$ & $0 \%$ & $0 \%$ \\
fev/17 & $5 \%$ & $82 \%$ & $0 \%$ & $0 \%$ & $14 \%$ & $0 \%$ & $0 \%$ \\
MÉDIA & $3 \%$ & $74 \%$ & $3 \%$ & $2 \%$ & $15 \%$ & $3 \%$ & $0 \%$ \\
\hline
\end{tabular}

Fonte: Autoria própria. 


\subsubsection{Construção dos cenários}

A partir dos dados históricos, inicialmente foram calculados a CFP da frota e seu gasto com combustível, o que será denominado de Cenário Real.

Para o objetivo deste trabalho, foi necessário projetar um cenário para cada mês em que a emissão de $\mathrm{CO}_{2 e}$ fosse minimizada, o qual foi denominado de "Cenário Verde". Analisando-se a aplicação da Eq. 02 ao longo de dado mês, observa-se que a distância é constante, de forma que o valor da variável CFP varia direta e proporcionalmente com o valor do FE $\mathrm{e}_{\mathrm{e}}$. Além disso, uma vez que toda a frota (com exceção da motocicleta) possui a mesma capacidade de carga (5 pessoas), considerou-se que quaisquer dos veículos disponíveis poderiam ter sido utilizados para realizarem as viagens.

Portanto, para se construir o Cenário Verde, supôs-se uma situação em que os trajetos tivessem sido realizados novamente, porém priorizando os veículos com menor $\mathrm{FE}_{\mathrm{e}} \mathrm{e}$, no caso dos veículos flex, utilizando o combustível com menor FE. Para isso, mês a mês os passos a seguir foram efetuados: Somaram-se as distâncias percorridas por cada veículo na situação real durante o mês em estudo. Em seguida, os maiores valores de distâncias percorridas foram atribuídos aos veículos com menores valores de $\mathrm{FE}_{\mathrm{e}}$. Por fim, foram calculados a CFP com a planilha de WRI (2016) e o custo financeiro total do mês com as equações abaixo:

$$
\begin{aligned}
& \text { CKR }=\text { Custo }_{\text {Combustivel }} * \frac{1}{A u t} \\
& \text { Custo }=\text { CKR } * \text { Dist }
\end{aligned}
$$

Nas quais:

- CKR = Custo do quilômetro rodado $(\mathrm{R} \$ / \mathrm{Km})$;

- Custo combustivel = Preço do litro do combustível utilizado pelo veículo em análise à época $(R \$ / L)$;

Os preços de cada combustível não constam na ferramenta, sendo encontrados no histórico disponibilizado mês a mês por município pela Agência Nacional do Petróleo, Gás Natural e Biocombustíveis (ANP) em seu site (ANP, 2017).

Por meio do Cenário Verde proposto, seria possível comparar assim o quão onerosa para a empresa seria a mudança da política de escolha dos veículos priorizando a minimização da CFP.

Contudo, considerou-se a possibilidade dos critérios aplicados pela empresa na situação real, embora visando a economia financeira, não necessariamente refletiam a situação de menor custo por viagem. Assim, a fim de promover melhor embasamento para a análise de onerosidade para a implantação de políticas de minimização de impactos ambientais, supôs-se um terceiro cenário, denominado de "Cenário Econômico", no qual os trajetos seriam realizados priorizando de fato os veículos com menor custo por quilômetro rodado. 
Para tanto, os mesmos passos para a construção do Cenário Verde foram seguidos, porém priorizando os veículos disponíveis com menores valores de CKR ao atribuir as distâncias percorridas no mês e o abastecimento com combustíveis mais econômicos nos flex.

Já as viagens realizadas pela motocicleta e pelos veículos pesados foram mantidas nos cenários alternativos, uma vez que sua capacidade de carga difere do restante da frota.

\subsubsection{Custo incremental do cenário verde}

É uma medida criada no decorrer deste trabalho a fim de relacionar a variação da CFP com o custo financeiro entre os cenários. Ela permite avaliar o custo financeiro que a empresa teria para deixar de emitir 1 tonelada de $\mathrm{CO}_{2 e}$ no período e cenário analisados, e é calculada seguindo a Eq. 05 abaixo:

$$
\text { CICV }=\frac{\text { Custo }_{\text {verde }}-\text { Custo }_{\text {cenario }}}{\left|C F P_{\text {verde }}-C F P_{\text {cenario }}\right|}
$$

Na qual:

- $\mathrm{ClCV}=$ Custo Incremental do Cenário Verde $\left(\mathrm{R} \$ / \mathrm{tCO}_{2 \mathrm{e}}\right)$;

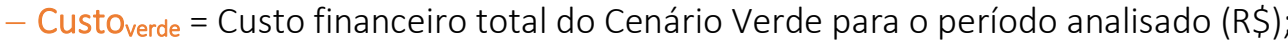

- Custo $_{\text {cenario }}=$ Custo financeiro total do Cenário comparado, podendo ser tanto o Econômico quanto o Real (R\$);

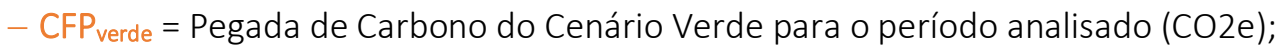

- CFP $_{\text {cenario }}=$ Pegada de Carbono do Cenário comparado, podendo ser tanto o Econômico quanto o Real $\left(\mathrm{CO}_{2 \mathrm{e}}\right)$.

Assim, quanto menor o valor do CICV, menor é o custo para a empresa minorar seus impactos ambientais no período analisado. Da mesma forma, valores altos de CICV indicam que a empresa elevaria muito seus custos para reduzir sua CFP.

Observando-se a Eq. 05, percebe-se que, para a comparação entre os cenários Verde e Econômico, o valor do CICV será sempre positivo, visto que o custo financeiro do cenário Econômico por definição será sempre o menor. Contudo, comparando os cenários Verde e Real, há também a possibilidade do CICV ser igual ou menor que zero. Nesses casos, o cenário Verde apresenta CFP e custo financeiro menores ou iguais ao cenário Real. Ou seja, o cenário Verde ou será mais economicamente vantajoso ou pelo menos não trará custos adicionais.

Contudo, nem sempre o valor do CICV pode ser facilmente considerado alto ou baixo, pois tal conclusão depende da ordem de grandeza dos custos dos cenários. Assim, torna-se necessário a introdução de uma segunda medida, a qual permita avaliar o quão impactante o aumento dos custos 
entre os cenários é para a empresa: o Incremento Percentual do Custo Verde, apresentada na seção a seguir.

\subsubsection{Incremento percentual do custo verde}

Este indicador permite avaliar a porcentagem de aumento no custo total da atividade durante o período considerado caso a empresa optasse por seguir o cenário verde, e é dado pela Equação 06 abaixo:

$$
I P C V=\frac{\text { Custo }_{\text {verde }}-\text { Custo }_{\text {cenario }}}{\text { Custo }_{\text {cenario }}}
$$

Na qual:

- IPCV = Incremento Percentual do Custo Verde.

Assim como para o CICV, o valor do IPCV sempre será positivo quando comparado com o cenário Econômico pelos motivos citados na Seção 3.2.4.

\subsubsection{Variação percentual da pegada}

É uma terceira medida utilizada nesse trabalho para avaliar os cenários, que expressa a relação entre a variação da CFP entre cenários e a quantidade de $\mathrm{CO}_{2 e}$ emitida no cenário mais poluente. A Eq. 07 explica como o cálculo dessa medida ocorre:

$$
V P P=\frac{C F P_{\text {verde }}-C F P_{\text {cenario }}}{C F P_{\text {cenario }}}
$$

Na qual:

- VPP = Variação Percentual da Pegada .

Como, por definição, o valor de CFPverde sempre será menor ou igual em relação a qualquer outro cenário do mesmo período, o valor de VPP sempre será negativo ou nulo.

\section{Resultados e discussão}

Visto que maior parte da frota é composta por veículos flex, os quais influenciam consideravelmente nos cálculos de CFP e custos financeiros, a consideração do combustível utilizado nos mesmos, ilustrada no Quadro 2, foi de grande importância para que os cenários refletissem a situação menos poluente ou mais econômica. 


\section{Quadro 2}

Combustível Utilizado Pelos Veículos Flex em Cada Cenário

\begin{tabular}{|lccc|}
\hline Mês & Verde & Econômico & Real \\
mar/16 & Etanol & Gasolina & Etanol \\
abr/16 & Etanol & Gasolina & Etanol \\
mai/16 & Etanol & Etanol & Etanol \\
jun/16 & Etanol & Etanol & Etanol \\
jul/16 & Etanol & Gasolina & Etanol \\
ago/16 & Etanol & Etanol & Etanol \\
set/16 & Etanol & Gasolina & Etanol \\
out/16 & Etanol & Gasolina & Etanol - Até $17 / 10$ \\
nov/16 & Etanol & Gasolina & Gasolina - Após $17 / 10$ \\
dez/16 & Etanol & Gasolina & Gasolina \\
jan/17 & Etanol & Gasolina & Gasolina \\
fev/17 & Etanol & Gasolina & Gasolina \\
\hline
\end{tabular}

Fonte: Autoria própria.

Como é possível observar, para o Cenário Verde foram escolhidos apenas o etanol como combustível pelo mesmo ter o menor valor de FEe, o que ocorre devido ao fato do etanol ser proveniente de biomassa, logo, de acordo com WRI (2008), as emissões de $\mathrm{CO}_{2}$ por sua queima são desconsideradas.

Para o cálculo do custo financeiro, utilizaram-se as equações Eq. 03 e Eq. 04. Os resultados podem ser encontrados na tabela 2 abaixo:

Tabela 2

Valores de CFP e Custos Financeiros das Atividades Logísticas nos Cenários Reais e Propostos

\begin{tabular}{|c|c|c|c|c|c|c|}
\hline \multicolumn{3}{|c|}{ Cenário Verde } & \multicolumn{2}{|c|}{ Cenário Econômico } & \multicolumn{2}{|c|}{ Cenário Real } \\
\hline Mês & $\begin{array}{c}\mathrm{CFP} \\
\left(\mathrm{tCO}_{2 \mathrm{e}}\right)\end{array}$ & Custo ( $R \$$ ) & $\begin{array}{c}\text { CFP } \\
\left(\mathrm{tCO}_{2 \mathrm{e}}\right)\end{array}$ & Custo (R\$) & $\begin{array}{c}\text { CFP } \\
\left(\mathrm{tCO}_{2 \mathrm{e}}\right)\end{array}$ & Custo (R\$) \\
\hline $\mathrm{mar} / 16$ & 0,372 & $15.047,82$ & 6,517 & $13.730,63$ & 0,599 & $14.966,94$ \\
\hline $\mathrm{abr} / 16$ & 0,261 & $14.011,67$ & 6,571 & $12.912,90$ & 0,309 & $14.007,16$ \\
\hline mai/16 & 0,207 & $11.387,91$ & 1,477 & $10.771,82$ & 0,865 & $10.868,43$ \\
\hline jun/16 & 0,323 & $13.562,78$ & 1,970 & $12.350,76$ & 0,336 & $12.598,31$ \\
\hline $\mathrm{jul} / 16$ & 0,255 & $15.122,03$ & 7,151 & $12.879,27$ & 0,291 & $14.831,35$ \\
\hline ago/16 & 0,226 & $12.529,64$ & 1,605 & $11.265,11$ & 0,246 & $11.545,79$ \\
\hline set/16 & 0,218 & $13.113,99$ & 5,376 & $12.019,13$ & 0,228 & $12.587,85$ \\
\hline out/16 & 0,218 & $14.249,35$ & 5,413 & $12.389,09$ & 2,667 & $12.979,64$ \\
\hline nov/16 & 0,203 & $11.391,65$ & 5,014 & $11.001,90$ & 5,128 & $11.258,36$ \\
\hline dez/16 & 0,164 & $9.461,04$ & 3,948 & $8.887,33$ & 3,991 & $8.983,90$ \\
\hline jan/17 & 0,213 & $11.959,78$ & 4,988 & $11.227,54$ & 5,080 & $11.437,41$ \\
\hline $\mathrm{fev} / 17$ & 0,243 & $14.433,97$ & 5,797 & $12.934,88$ & 5,950 & $13.281,47$ \\
\hline Total & 2,902 & $156.271,64$ & 55,826 & $142.370,37$ & 25,691 & $149.346,61$ \\
\hline
\end{tabular}

Fonte: Autoria própria. 
Na tabela 2 e nas seguintes, os valores foram coloridos em uma escala variando do vermelho ao verde para fins de ordenação ao longo de cada coluna. Para as variáveis relacionadas à Pegada de Carbono (CFP, $\triangle \mathrm{CFP}, \mathrm{VPP}, \mathrm{CICV}$ ), os valores que indicam menores impactos ambientais ou maior redução destes possuem matiz mais próximo da cor verde. Para as variáveis referentes apenas à indicadores econômicos (Custo, $\triangle$ Custo, IPCV), a proximidade da cor verde indica menores gastos financeiros. Ressalta-se que a escala de cor não visa estabelecer limiares de valores, com os marcados em verde sendo aceitáveis e os marcados em vermelho, não aceitáveis. Em vez disso, as cores visam auxiliar na identificação dos maiores e menores valores ao longo do período para cada variável.

Observa-se pela tabela 2 que na maioria dos meses considerados há uma grande variação nos valores de CFP entre os cenários Verde e Real e entre Verde e Econômico, porém não muito significativa nos valores dos custos. Para expressar essa relação entre redução de CFP e aumento dos custos; o quão impactante tal aumento seria no caixa da empresa; e o quão relevante foi a diminuição da CFP total, calcularam-se o CICV, IPCV e VPP para cada mês, cujos resultados encontram-se nas tabelas 3 e tabela 4 abaixo:

Tabela 3

Variação dos Parâmetros Partindo-se do Cenário Real para o Verde

\begin{tabular}{lccccc}
\hline \multicolumn{1}{c}{ Mês } & $\begin{array}{c}\Delta C F P \\
\left(\mathrm{CO}_{2 \mathrm{e}}\right)\end{array}$ & VPP & $\begin{array}{c}\Delta \text { Custo } \\
(\mathrm{R} \$)\end{array}$ & $\begin{array}{c}\mathrm{CICV} \\
\left(\mathrm{RS} / \mathrm{CO}_{2 \mathrm{e}}\right)\end{array}$ & IPCV \\
\hline $\mathrm{mar} / 16$ & $-0,227$ & $-38 \%$ & 80,88 & 356,63 & $0,540 \%$ \\
abr/16 & $-0,048$ & $-15 \%$ & 4,51 & 94,69 & $0,03 \%$ \\
mai/16 & $-0,658$ & $-76 \%$ & 519,48 & 789,29 & $4,78 \%$ \\
jun/16 & $-0,013$ & $-4 \%$ & 964,47 & $73.011,72$ & $7,66 \%$ \\
jul/16 & $-0,036$ & $-12 \%$ & 290,68 & $8.018,62$ & $1,96 \%$ \\
ago/16 & $-0,020$ & $-8 \%$ & 983,86 & $48.440,34$ & $8,52 \%$ \\
set/16 & $-0,009$ & $-4 \%$ & 526,14 & $55.409,55$ & $4,18 \%$ \\
out/16 & $-2,449$ & $-92 \%$ & $1.269,71$ & 518,46 & $9,78 \%$ \\
nov/16 & $-4,926$ & $-96 \%$ & 133,29 & 27,06 & $1,18 \%$ \\
dez/16 & $-3,827$ & $-96 \%$ & 477,14 & 124,69 & $5,31 \%$ \\
jan/17 & $-4,867$ & $-96 \%$ & 522,38 & 107,32 & $4,57 \%$ \\
fev/17 & $-5,708$ & $-96 \%$ & $1.152,50$ & 201,92 & $8,68 \%$ \\
Total & $-22,788$ & $-89 \%$ & $6.925,03$ & 303,89 & $4,64 \%$ \\
\hline
\end{tabular}

Fonte: Autoria própria.

Como pode-se observar na tabela 3, durante a maioria dos meses há um padrão: o CICV possui um valor baixo quando comparados às colunas Custo custa na tabela 2 (média de $R \$ 303,89$ ao longo de 1 ano); as porcentagens na coluna do IPCV e os valores da coluna $\triangle$ Custo revelam que o aumento no custo total caso a empresa tivesse optado por seguir a alternativa menos poluente seria de $R \$ 6.925,03$ ao todo, ou seja, $4,64 \%$ do custo já executado para a atividade; por fim, as colunas $\triangle C F P$ e VPP 
expressam a melhora do ponto de vista ambiental que tal priorização promoveria, reduzindo 22,788 toneladas de $\mathrm{CO}_{2 \mathrm{e}}$ ao longo de 1 ano, o equivalente a deixar de emitir $89 \%$ do que foi lançado na atmosfera.

Alguns meses, porém, chamam a atenção devido aos seus valores extremos ou atípicos do restante da tabela 3, como o período entre junho e setembro de 2016. Tomando como exemplo o mês de setembro, são observados grandes valores de CICV (R\$ 55.409,55). Analisando a coluna isoladamente, poder-se-ia concluir erroneamente que o custo para reduzir o nível de poluição atmosférica pela frota torna tal atitude financeiramente inviável. Contudo, tal fato exemplifica a importância de se analisar os indicadores em conjunto: observando-se a Eq. 05, nota-se que, quanto menor a variação da CFP entre cenários, maior será o valor do CICV, como de fato ocorreu nesse período, quando a CFP no cenário Verde seria apenas 0,009 $\mathrm{tCO}_{2 \mathrm{e}}$ menor em relação ao Real, representando $4 \%$ do valor emitido na situação real. Observando-se as colunas $\triangle$ Custo e IPCV, percebese que, para não degradar a atmosfera nessa quantidade de poluentes, o custo investido seria de $R \$$ 526,14, equivalente a 4,18\% do custo já decorrido no mesmo período.

Caso semelhante ocorre no mês de abril, quando os valores de $\triangle$ CFP e VPP indicam baixa melhora ambiental entre a variação dos cenários $\left(0,048 \mathrm{tCO}_{2 \mathrm{e}}\right)$. Porém, o custo para se promover tal melhoria totaliza $\mathrm{R} \$ 4,51$, ou $0,03 \%$ do custo Real.

Tabela 4

Variação dos Parâmetros Partindo-se do Cenário Econômico Para o Verde

\begin{tabular}{lccccc}
\hline \multicolumn{1}{c}{ Mês } & $\begin{array}{c}\Delta C F P \\
\left(\mathrm{CO}_{2 \mathrm{e}}\right)\end{array}$ & $\mathrm{VPP}$ & $\begin{array}{c}\Delta \text { Custo } \\
(\mathrm{R} \$)\end{array}$ & $\begin{array}{c}\mathrm{ClCV} \\
\left(\mathrm{RS} / \mathrm{CO}_{2 \mathrm{e}}\right)\end{array}$ & IPCV \\
\hline mar/16 & $-6,14$ & $-94 \%$ & $1.317,19$ & 214,36 & $9,59 \%$ \\
\hline abr/16 & $-6,31$ & $-96 \%$ & $1.098,78$ & 174,15 & $8,51 \%$ \\
mai/16 & $-1,27$ & $-86 \%$ & 616,09 & 485,30 & $5,72 \%$ \\
jun/16 & $-1,65$ & $-84 \%$ & $1.212,02$ & 735,59 & $9,81 \%$ \\
jul/16 & $-6,90$ & $-96 \%$ & $2.242,76$ & 325,23 & $17,41 \%$ \\
ago/16 & $-1,38$ & $-86 \%$ & $1.264,54$ & 916,56 & $11,23 \%$ \\
set/16 & $-5,16$ & $-96 \%$ & $1.094,86$ & 212,27 & $9,11 \%$ \\
out/16 & $-5,19$ & $-96 \%$ & $1.860,26$ & 358,11 & $15,02 \%$ \\
nov/16 & $-4,81$ & $-96 \%$ & 389,74 & 81,00 & $3,54 \%$ \\
dez/16 & $-3,78$ & $-96 \%$ & 573,71 & 151,63 & $6,46 \%$ \\
jan/17 & $-4,78$ & $-96 \%$ & 732,24 & 153,34 & $6,52 \%$ \\
fev/17 & $-5,55$ & $-96 \%$ & $1.499,08$ & 269,92 & $11,59 \%$ \\
Total & $-52,92$ & $-95 \%$ & $13.901,28$ & 262,67 & $9,76 \%$ \\
\hline
\end{tabular}

Fonte: Autoria própria.

Conforme explicado na seção 3.2, o cenário Econômico foi projetado com base na suposição de que os critérios adotados pela empresa para seleção dos veículos e dos combustíveis para os flex não 
refletissem de fato a alternativa com menor custo, o que é confirmado comparando-se a coluna dos custos entre os cenários Real e Econômico da tabela 2.

As colunas $\triangle$ CFP e VPP da tabela 4 expressam que, em todos os meses, a variação da CFP foi consideravelmente alta, implicando em melhorias significativas ao meio ambiente ao variar os cenários, totalizando uma redução de 52,92 $\mathrm{tCO}_{2 \mathrm{e}}$ ao longo do ano, ou seja, 95\% da quantidade que seria emitida. Os menores valores do CICV também indicam uma grande melhoria ambiental em decorrência do aumento dos gastos com combustível, de forma que, no fim da série temporal, o desembolso para se economizar $1 \mathrm{tCO}_{2 \mathrm{e}}$ é de $\mathrm{R} \$ 262,67$.

Os incrementos de custo são maiores quando se implementa o cenário Verde a partir do Econômico do que quando se parte do Real, indicando a necessidade de se desembolsar mais nessa primeira variação de cenários, porém com uma redução mais significativa da CFP.

Além dos cálculos das variáveis acima apresentados e suas respectivas análises, neste trabalho também se desenvolveu um fluxograma para caso a empresa decida, com base nos dados supracitados, optar por uma logística mais verde priorizando os veículos menos poluentes para as atividades do setor Frota Baixa, o qual segue na figura 2. 
Figura 2

Modelo Para seleção de Veículos Para o Setor Frota Baixa Adotar o Cenário Verde

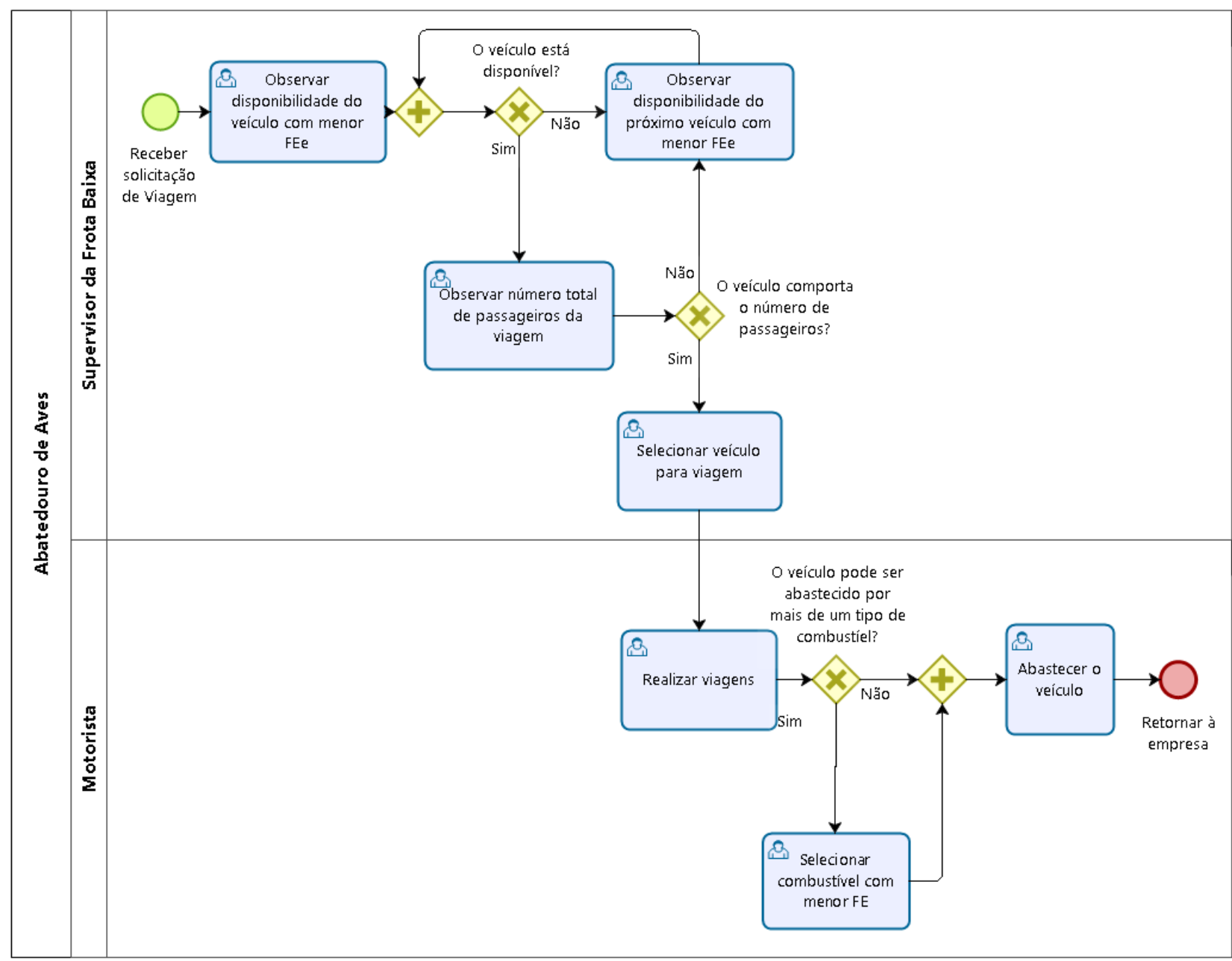

Fonte: Autoria própria.

A partir dos resultados apresentados, percebe-se que, pelo menos neste caso, corresponde à realidade a preocupação das empresas de que a redução dos impactos ambientais provoque aumento de custos financeiros, conforme apontado por Walker, Di Sisto e Mc Bain (2008). Contudo, os resultados deste caso também mostram que o aumento nos custos decorrente da escolha de alternativas que proporcionem menores impactos ambientais pode ser proporcionalmente bem menor do que os benefícios ambientais.

\section{Conclusões}

Por meio deste trabalho foi possível mensurar o impacto financeiro para a empresa minimizar a Pegada de Carbono na alocação de veículos da frota baixa durante um período de 12 meses. Isso foi feito a partir da comparação dos gastos em dois cenários hipotéticos com relação aos gastos efetivamente ocorridos durante o período de análise (cenário Real). O primeiro cenário hipotético, o 
"cenário Verde" prioriza a redução da poluição atmosférica por meio da seleção de combustíveis e veículos que emitem menos GEE. O segundo cenário hipotético, o "cenário Econômico" prioriza a redução dos custos com combustível.

Caso o cenário verde fosse adotado pela empresa, haveria uma redução total de $22,788 \mathrm{tCO}_{2 \mathrm{e}}$ ou $89 \%$ do que foi emitido no cenário Real, com um aumento de $\mathrm{R} \$ 6.925,03$ ou 4,64\% dos custos totais com combustível. Já quando se compara o cenário Verde com o cenário Econômico, a CFP do primeiro seria $52,92 \mathrm{tCO}_{2 \text { e }}$ ou $95 \%$ menor do que a do segundo, com um aumento de $\mathrm{R} \$ 13.901,28$ ou 9,76\% nos custos com abastecimento.

Também se sugeriu uma sequência operacional para que a seleção do veículo de cada futura viagem privilegie a minimização dos impactos ambientais (avaliados em termos do indicador ambiental "pegada de carbono") decorrentes da mesma. Caberia à empresa decidir se tais gastos incrementais seriam aceitáveis para os benefícios ambientais esperados.

Assim, a mensuração das emissões em frotas particulares e o aumento em seus custos de operações decorrentes da redução dessas emissões pode auxiliar na tomada de decisão em se implantar políticas que priorizem a minimização de impactos ambientais, de forma a contribuir para uma Logística Verde.

\section{Referências}

Agência Nacional do Petróleo, Gás Natural e Biocombustíveis. (2017) Série histórica do levantamento de preços e de margens de comercialização de combustíveis. Brasil. Disponível em: < http://www.anp.gov.br/wwwanp/precos-e-defesa/234-precos/levantamento-de-precos/868serie-historica-do-levantamento-de-precos-e-de-margens-de-comercializacao-decombustiveis >. Acesso em: 12 set. 2017.

Ballou, R. H. (2006). Gerenciamento da cadeia de suprimentos/logística empresarial. (5a. ed.). Porto Alegre: Bookman.

Borucke, M., Moore, D., Cranston, G., Gracey, K., Iha, K., Larson, J., Lazarus, E., Morales, J. C., Wackernagel, M. \& Galli, A. (2013). Accounting for demand and supply of the biosphere's regenerative capacity: The National Footprint Accounts' underlying methodology and framework. Ecological Indicators, 24, 518-533. https://doi.org/10.1016/j.ecolind.2012.08.005

Bullard, C. W., Penner, P. S. \& Pilati, D. A. (1978) Net energy analysis: Handbook for combining process and input-output analysis. Resources and Energy, 1(3), 267-313, 1978. http://dx.doi.org/10.1016/0165-0572(78)90008-7.

Carbon Trust (2012). Carbon footprinting - The next step to reduce your emissions. Reino Unido.

Donato, V. (2008). Logística verde. Rio de Janeiro: Ciência Moderna Ltda. 
Duez, B. (2016). Towards a substantially lower fuel consumption freight transport by the development of an innovative low rolling resistance truck tyre concept. Transportation Research Procedia, 14, 1051-1060. https://doi.org/10.1016/J.TRPRO.2016.05.175

Global Footprint Network (2017). Climate change. Disponível em: < http://www.footprintnetwork.org/our-work/climate-change/ >. Acesso em 4 jul. 2017.

Hao, H., Geng, Y. \& Sarkis, J. (2016). Carbon footprint of global passenger cars: scenarios through 2050. Energy, 101, 121-131. https://doi.org/10.1016/j.energy.2016.01.089

Intergovernmental Panel On Climate Change (2014). Climate change 2014: Synthesis report. Contribution of working groups I, II and III to the Fifth Assessment Report of the Intergovernmental Panel on Climate Change [Core Writing Team, R.K. Pachauri and L.A. Meyer (eds.)]. Genebra, Suíça.

Intergovernmental Panel On Climate Change (2006). National Greenhouse Gas Inventories Programme. 2006 IPCC guidelines for National Greenhouse Gas Inventories. Hayama, JP: IGES, 2(3). Disponível em: < http://www.ipccnggip.iges.or.jp/public/2006gl/pdf/2_Volume2/V2_3_Ch3_Mobile_Combustio n.pdf >. Acesso em: 23 abr. 2017.

ISO (2013). ISO 14067:2013 Greenhouse gases - Carbon footprint of products - Requirements and guidelines for quantification and communication. International Organization for Standardization.

Kellner, F. \& Igl, Johannes (2015). Greenhouse gas reduction in transport: analyzing the carbon dioxide performance of different freight forwarder networks. Journal of Cleaner Production, 99, 177191. https://doi.org/10.1016/j.jclepro.2015.03.026

Mckinnon, A., Browne, M., Whiteing, A. \& Piecyk, M. (2010). Green logistics: improving the environmental sustainability of logistics. Londres: Kogan Page.

Meixell, M. J. \& Norbis, M. (2008). A review of the transportation mode choice and carrier selection literature. The International Journal of Logistics Management, 19(2), 183-211. https://doi.org/10.1108/09574090810895951

Ministério da Agricultura, Pecuária e Abastecimento. Gabinete da Ministra. (2015). Portaria no. 75, de 5 de março de 2015. Diário Oficial da União, Brasília, DF. Seção 1, 17. Disponível em: < http://pesquisa.in.gov.br/imprensa/jsp/visualiza/index.jsp?data=06/03/2015\&jornal=1\&pagin $a=17 \&$ totalArquivos=200 >. Acesso em: 12 set. 2017.

Ministério do Meio Ambiente (2014). 2o Inventário nacional de emissões atmosféricas por veículos automotores rodoviários. Brasília, DF, Secretaria de Mudanças Climáticas e Qualidade Ambiental, Diretoria de Mudanças Climáticas. Disponível em: < http://www.mma.gov.br/images/arquivo/80060/Inventario_de_Emissoes_por_Veiculos_Rodo viarios_2013.pdf >. Acesso em: 23 abr. 2017.

Murphy, P. R., Poist, R. F. \& Braunschweig, C. D. (1995). Role and relevance of logistics to corporate environmentalism. International Journal of Physical Distribution \& Logistics Management, 25(2), 5-19. https://doi.org/10.1108/09600039510083916 
Quiumento, F. (2011). Logística Verde: Uma nova visão para a logística com atividade humana integrada ao ambiente. Disponível em: < http://knowledgeispowerquiumento.wordpress.com/article/logistica-verde-2tlel7k7dcy4s-90/ >. Acesso em: 06/04/2013.

Saenz, J., Figliozzi, M. \& Faulin, J (2016). Assessment of the carbon footprint reductions of tricycle logistics services. Transportation Research Record: Journal of the Transportation Research Board, 2570, 48-56. https://doi.org/10.3141/2570-06

Santana, M., \& Oiko, O. T. (2019). Pegada de Carbono: Em busca de definição e método para uma logística verde. Gepros: Gestão da Produção, Operações e Sistemas, 14(4), 197. https://doi.org/10.15675/gepros.v14i4.2334

Santos, J., Bortolon, K., Chiroli, D. \& Oiko, O. (2015). Logística verde: conceituação e direcionamentos para aplicação. Electronic Journal of Management, Education and Environmental Technology (REGET), 19(2), 314-331. https://doi.org/10.5902/2236117015912

Seuring, S. \& Müller, M. (2008). From a literature review to a conceptual framework for sustainable supply chain management. Journal of Cleaner Production, 16(15), 1699-1710. https://doi.org/10.1016/j.jclepro.2008.04.020

Silva, A. L., Ferreira, J. C., \& Steiner, M. T. A. (2019). Abordagem de roteamento de veículos com procedimentos exatos e heurísticos sob uma óptica ambiental: um estudo de caso. Exacta, 17(1), 171-187. https://doi.org/10.5585/exactaep.v17n1.8171

Silva, F., Vaz, C., \& Lezana, A. (2020). Green Supply Chain Management e logística reversa: uma análise sistemática dos artigos. Exacta, 18(4), 686-707. https://doi.org/10.5585/exactaep.v18n4.8457

The British Standards Institution. (2011). Publicly Available Specification PAS 2050: 2011 - Specification for the assessment of the life cycle greenhouse gas emissions of goods and services. Londres. Disponível em: < http://shop.bsigroup.com/upload/shop/download/pas/pas2050.pdf >. Acesso em: 3 jul. 2017.

Walker, H., Di Sisto, L. \& Mcbain, D. (2008). Drivers and barriers to environmental supply chain management practices: Lessons from the public and private sectors. Journal of Purchasing and Supply Management, 14(1), 69-85. https://doi.org/10.1016/j.pursup.2008.01.007

Wiedman, T. \& Minx, J. (2008). A definition of "Carbon Footprint". ISA UK Research Report 07-01. Ecological Economics Research Trends (Cap. 1, pp. 1-11). USA: Nova Science Publishers. Disponível em: < https://www.novapublishers.com/catalog/product_info.php?products_id=5999 >. Acesso em: 27 ago. 2016.

Winsemius, P. \& Guntram, U. (1992) Responding to the environmental challenge. Business Horizons, 35(2), 12-20. https://doi.org/10.1016/S0007-6813(05)80188-0

Wolf, C. \& Seuring, S. (2010). Environmental impacts as buying criteria for third party logistical services. International Journal of Physical Distribution \& Logistics Management, 40(1/2), 84102. https://doi.org/10.1108/09600031011020377

World Resource Institute (2008). Especificações do programa brasileiro GHG Protocol: contabilização, quantificação e publicação de inventários corporativos de emissões de gases de efeito estufa. 
(2a ed.). Disponível em: < https://s3-sa-east-

1.amazonaws.com/arquivos.gvces.com.br/arquivos_ghg/152/especificacoes_pb_ghgprotocol. pdf >. Acesso em: 23 abr. 2017.

World Resource Institute (2016). Ferramenta de cálculo. Disponível em: <

http://www.ghgprotocolbrasil.com.br/ferramenta-de-calculo >. Acesso em: 28 ago. 2017.

Zabeo, A., Bellio, C., Pizzol, L., Giubilato, E.\& Semenzin, E. (2017). Carbon footprint of municipal solid waste collection in the Treviso area (Italy). Environmental Engineering and Management Journal, 16(8), 1781-1787. Disponível em: < http://eemj.eu/index.php/EEMJ/article/view/3350 >. Acesso em: 07 apr. 2018. 